\title{
Ablative radiation therapy for hepatocellular carcinoma is associated with reduced treatment- and tumor-related liver failure and improved survival
}

\author{
Lara Hilal $^{1}$, Marsha Reyngold ${ }^{1}$, Abraham J. Wu ${ }^{1}$, Abdallah Araji ${ }^{2}$, Ghassan K. Abou-Alfa, \\ William Jarnagin ${ }^{5}$, James J. Harding ${ }^{3,4}$, Maya Gambarin ${ }^{3,4}$, Imane El Dika ${ }^{3,4}$, Paul Brady ${ }^{1}$, John Navilio ${ }^{6}$, \\ Sean L. Berry ${ }^{6}$, Jessica Flynn ${ }^{7}$, Zhigang Zhang $^{7}$, Richard Tuli ${ }^{1}$, Melissa Zinovoy ${ }^{1}$, Paul B. Romesser ${ }^{1}$, \\ John J. Cuaron ${ }^{1}$, Christopher H. Crane ${ }^{1}$, Carla Hajj ${ }^{1}$
}

${ }^{1}$ Department of Radiation Oncology, Memorial Sloan Kettering Cancer Center, New York, NY, USA; ${ }^{2}$ Department of Radiology, Memorial Sloan Kettering Cancer Center, New York, NY, USA; ${ }^{3}$ Department of Medicine, Memorial Sloan Kettering Cancer Center, New York, NY, USA; ${ }^{4}$ Department of Medicine, Weill Cornell Medical College, New York, NY, USA; ${ }^{5}$ Department of Surgery, Memorial Sloan Kettering Cancer Center, New York, NY, USA; ${ }^{6}$ Department of Medical Physics, Memorial Sloan Kettering Cancer Center, New York, NY, USA; ${ }^{7}$ Department of Epidemiology and Biostatistics, Memorial Sloan Kettering Cancer Center, New York, NY, USA

Contributions: (I) Conception and design: C Hajj, M Reyngold, L Hilal, CH Crane; (II) Administrative support: P Brady; (III) Provision of study materials or patients: M Reyngold, AJ Wu, GK Abou-Alfa, W Jarnagin, JJ Harding, M Gambarin, I El Dika, R Tuli, M Zinovoy, PB Romesser, JJ Cuaron, CH Crane, C Hajj; (IV) Collection and assembly of data: L Hilal, J Navilio, P Brady, SL Berry, A Araji; (V) Data analysis and interpretation: JF, ZZ, L Hilal, M Reyngold, AJ Wu, GK Abou-Alfa, W Jarnagin, JJ Harding, M Gambarin, I El Dika, R Tuli, M Zinovoy, PB Romesser, JJ Cuaron, CH Crane, C Hajj; (VI) Manuscript writing: All authors; (VII) Final approval of manuscript: All authors.

Correspondence to: Carla Hajj, MD. Department of Radiation Oncology, Memorial Sloan Kettering Cancer Center, New York, NY, USA. Email: Hajjc@mskcc.org.

Background: More than $70 \%$ of patients with hepatocellular carcinoma (HCC) are not candidates for curative therapy or recur after curative-intent therapy. There is growing evidence on the use of ablative radiation therapy (RT) for liver tumors. We aimed to analyze outcomes of HCC patients treated with conventional versus ablative RT.

Methods: We retrospectively analyzed medical records of HCC patients treated with liver RT from 2001 to 2019. We defined ablative RT as biologically effective dose (BED) $\geq 80$ Gy. RECIST 1.1 was used to define early responses at 3-6 months after RT, and local control (LC) at last follow-up (FU). Data was analyzed using Fisher exact test, Kaplan-Meier, cumulative incidence rates, Cox proportional hazards model and FineGray competing risks.

Results: Forty-five patients were identified, of whom 14 (31.1\%) received ablative RT using a stereotactic technique. With median FU of survivors of 10.1 months, 1-year cumulative incidence of LC was $91.7 \%$ for ablative and $75.2 \%$ for BED <80 Gy. At early FU, patients treated with ablative RT had better responses compared to BED $<80 \mathrm{~Gy}$, with $7 \%$ progressing versus $19 \%$, and $21.4 \%$ with complete response versus none $(\mathrm{P}=0.038)$. On univariate analysis (UVA), Child-Pugh (CP) score [hazard ratio (HR): 3 for CP-B, HR: 16 for CP-C] and BED (HR: 7.69 for BED <80 Gy) correlated with deterioration of liver function, leading to liver failure. Most liver failure cases were due to disease progression. No RT-related liver failure occurred in the ablative RT group. On UVA, only BED $\geq 80$ Gy was associated with improved overall survival (OS) (HR: 0.4; $\mathrm{P}=0.044$ ). Median OS (mOS) and 1-year OS were 7 months and 35\% respectively for BED $<80$ Gy compared to 28 months and $66 \%$ for BED $\geq 80$ Gy. No grade 3+ bowel toxicity was reported in either group. Conclusions: Greater than $90 \%$ LC was achieved after stereotactic ablative RT, which was associated with minimized tumor- and treatment-related liver failure and improved survival for highly selected inoperable HCC patients.

Keywords: Hepatocellular carcinoma (HCC); ablative radiation therapy; stereotactic radiation therapy; liver failure 
Submitted Mar 03, 2021. Accepted for publication Jun 08, 2021.

doi: 10.21037/jgo-21-116

View this article at: https://dx.doi.org/10.21037/jgo-21-116

\section{Introduction}

Liver cancer incidence is increasing in the United States, with doubling annual HCC-related death (1). Hepatocellular carcinoma (HCC) is the most common primary liver malignancy. Although surgery, radiofrequency ablation or liver transplantation are potentially curative for nonmetastatic HCC patients with intact liver function, the majority of patients are either medically inoperable, have unresectable disease (2), recur after therapy with curative intent, or have significant underlying liver disease (3). Patients with inoperable advanced HCC have a poor prognosis with median survival of around 14-16 months $(4,5)$. The majority of patients with advanced liver tumors die of liver failure due to cirrhosis or intrahepatic progression (5).

Locoregional treatment options for inoperable HCC include a variety of arterially directed embolization and radiation therapy (RT). There is growing evidence that supports the use of ablative RT for the treatment of liver tumors, including HCC (5-8). With the advancement of radiation techniques, respiratory motion management, and image guidance, there has been an improvement in the therapeutic ratio of treating liver tumors. In contrast to other locoregional modalities, RT is not as limited by tumor size, presence of vascular thrombus, or proximity to the biliary tree, liver hilum, and portal vein. Stereotactic body radiation therapy (SBRT) using 3-6 fractions has been shown to be effective in several retrospective series and phase I/II clinical trials with local control (LC) of $65-100 \%$ at 1 year (6). However, the main challenge is usually delivering high enough doses to ablate the tumor without causing serious adverse effects to the organs at risk (OAR) [mainly the gastrointestinal (GI) tract and the normal liver parenchyma], and most importantly, preserving liver function in cases of large tumors. This is where a more protracted hypofractionated ablative RT course can be more safely delivered (5).

To date, the majority of published studies on the use of hypofractionated ablative doses for HCC, using 10 or more fractions, have used protons (8-11). Protons spare liver parenchyma because of near-zero exit dose. Photonbased hypofractioned ablative RT is able to spare adjacent luminal organs better than protons and has been shown to be effective in the treatment of liver tumors such as HCC, liver metastases and intrahepatic cholangiocarcinoma (IHCC) $(7,12,13)$.

There is no clear consensus regarding the optimal dose fractionation for photon-based RT for HCC. Many clinicians wish to give 5 fraction regimens but are reluctant to give ablative doses due to safety concerns.

In this study, we aimed to retrospectively analyze outcomes of HCC patients treated at a single institution with conventional versus ablative photons RT.

We present the following article in accordance with the STROBE reporting checklist (available at https://dx.doi. org/10.21037/jgo-21-116).

\section{Methods}

\section{Study design and participants}

This is a single institution retrospective study. We identified all patients who received liver-directed RT for radiographically or pathologically diagnosed HCC from the institutional database, from January 1, 2000 to July 1, 2019 ( $n=45)$.

Patient and tumor characteristics, in addition to details of systemic and liver-directed therapies, were collected from the electronic medical records. Child-Pugh (CP) score was calculated (14) at the time of RT and then at each follow-up to assess for a change.

The study was conducted in accordance with the Declaration of Helsinki (as revised in 2013). The study was approved by institutional board of Memorial Sloan Kettering Cancer Center (MSKCC), New York (IRB 16370) and individual consent for this retrospective analysis was waived.

\section{RT details}

In 2016, we started using ablative radiation doses with a stereotactic hypofractionated technique for the treatment of localized unresectable HCC. HCC cases are discussed at a multidisciplinary tumor board prior to referral for ablative RT. Biologically effective dose (BED), a measure of the true biological dose delivered by a particular combination of dose per fraction and total dose delivered to a particular tissue, was 
Table 1 Dose-volume constraints for ablative liver radiation therapy

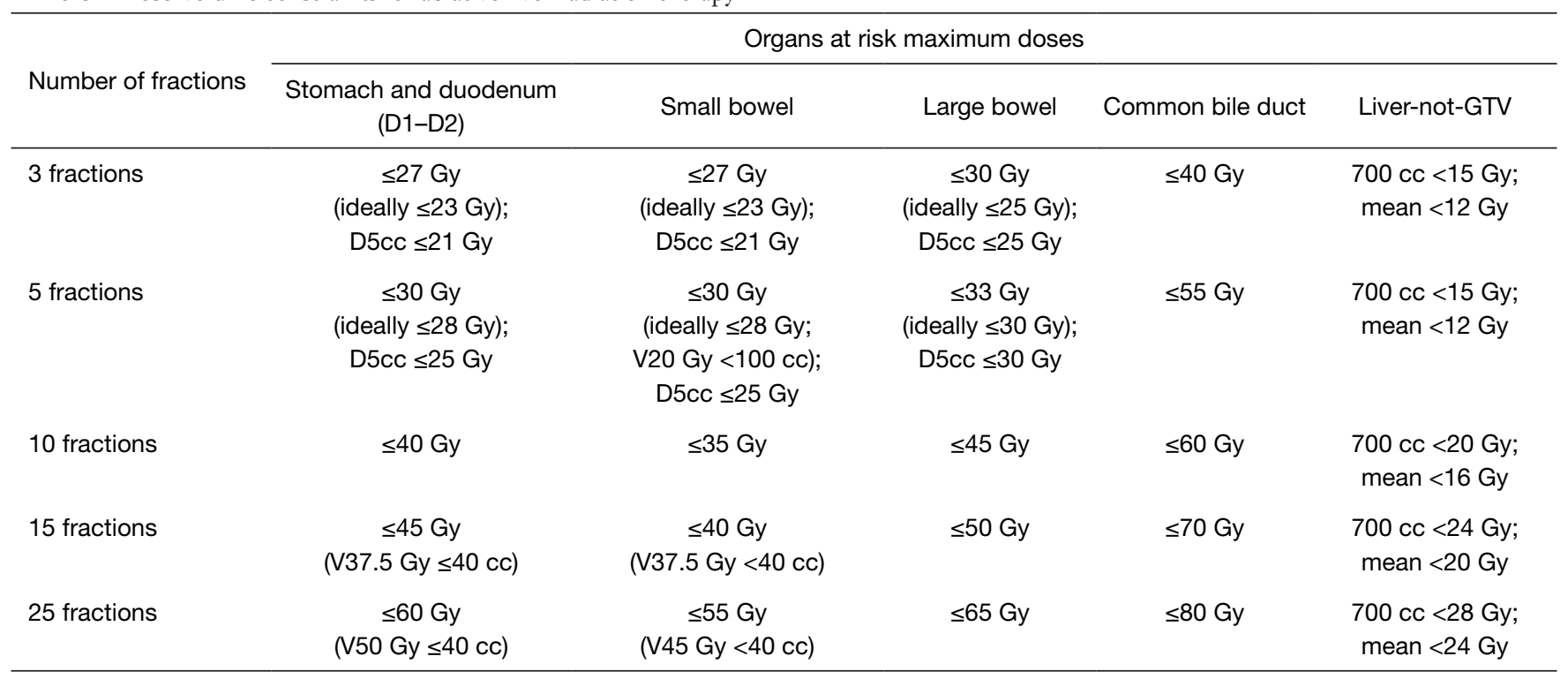

Doses are "maximum doses" unless specified otherwise. GTV, gross tumor volume.

calculated for all patients. This was done using an alpha/beta ratio of $10 \mathrm{~Gy}$. Ablative RT is defined in this study as BED $\geq 80$ Gy. BED $\geq 80$ Gy was chosen as an ablative RT dose given that HCC is a radiosensitive tumor and extrapolated from the study by Tao et al. on IHCC, where they specified a BED of 80.5 Gy as ablative (7).

For ablative doses, most patients had fiducials placed prior to simulation and treatment. All patients underwent planning computed tomography (CT) simulation scans in the treatment position with intravenous contrast. Gross tumour volume (GTV) was contoured on the contrastenhanced simulation scans. Deep inspiration breath hold technique was preferably used for motion management. For those patients unable to comply with DIBH, a 4DCT was used and an internal target volume (ITV) was created. For OAR, mainly the stomach, duodenum, and small bowel, we created a $3-5 \mathrm{~mm}$ expansion of adjacent OAR to create a planning risk volume (PRV). The PRV was then subtracted from the high-dose volume to ensure protection of the OAR. Planning target volume (PTV) was created by expanding GTV/ITV by $5 \mathrm{~mm}$ subtracted by the OAR expansion. In cases with more than one dose level, CTV low dose was a 1-cm expansion on GTV/ITV and PTV low dose was CTV low dose $+5 \mathrm{~mm}$. CTV high dose was 5 -mm expansion on GTV/ITV subtracted by OAR expansion and PTV high dose $=$ CTV high dose.
The most commonly used dose fractionation regimens in this cohort were $50 \mathrm{~Gy}$ in $5(\mathrm{BED}=100 \mathrm{~Gy})$ and $60 \mathrm{~Gy}$ in 15 fractions (BED =84 Gy). Prescription dose was the dose prescribed to the periphery of the PTV with the following aims: GTV: V100\% of prescription dose $=90 \%$ in an ideal scenario, this can go down to V100 (GTV) $=80 \%$ and PTV D95\% $\geq 90 \%$ of the prescription dose. The fractionation scheme used was based on the size of the tumor and its proximity to OAR. As an institutional RT guideline for treating liver tumors, doses of 50-60 Gy in 5 fractions, 45-75 Gy in 3 fractions, or 60-70 Gy in 10 are usually used for smaller peripheral lesions, away from the GI tract. For large $(>5 \mathrm{~cm}$ ) or central (within $2 \mathrm{~cm}$ from the porta hepatis) tumors, two or three dose levels are used as follows: $37.5 / 67.5$ Gy or 37.5/60 Gy in 15 fractions with an optional 90 Gy hotspot created by contracting the PTV67.5 Gy by $0.5-1 \mathrm{~cm}$, if away from the biliary tree. If the liver or GI dose constraints could not be met with the above fractionation, 45/75 Gy in 25 fractions is used with an optional 100 Gy hotspot created by contracting PTV75 Gy by $0.5-1 \mathrm{~cm}$, also if far enough from the proximal biliary tree. Table 1 summarizes the dose constraints for ablative RT.

RT was delivered using $6 \mathrm{MV}$ photon beam intensity modulated radiation therapy (IMRT) with image verification. 


\section{Outcomes and toxicities}

Patients were followed with a CT scan 2-3 months after completion of RT and every 6 months thereafter. All included patients had follow-up multiphasic CT scans. Patients were followed until progression, or until being censored for those whose disease remained controlled at the time of last followup. LC was determined by a single radiologist (A Araji) using RECIST version 1.1 criteria (15). RECIST version 1.1 was used to assess whether lesions showed partial response, complete response, stable disease, or disease progression. Lesions that showed no progression of disease were considered to be locally controlled. LC and overall survival (OS) were both calculated from the time of the end of RT treatment.

Toxicity data collected included GI ulceration or hemorrhage, biliary stenosis or cholangitis, ascites, as well as liver decompensation. Common terminology criteria for adverse events (CTCAE) version 5 was used for grading (16). Liver failure was defined as deterioration of liver function leading to a non-transient change in CP score and ultimately leading to death within 3 months. CP score was calculated at the start of RT and followed until last follow-up. The median follow-up for the CP score was 4 months with a range of 1-39 months.

\section{Statistical analysis}

Patients, tumor and treatment characteristics were summarized using median and range or frequency and percentages for the group of patients who received BED $<80$ Gy and for the patients who received ablative RT.

OS and progression-free survival (PFS) were estimated using Kaplan-Meier curves for the whole cohort, and stratified by BED. Differences in RECIST early response between patients treated with BED <80 Gy and ablative RT were analyzed using Fisher exact test. LC, distant progression, and liver failure were estimated using cumulative incidence rates, considering death as a competing risk. Univariate analysis using Cox proportional hazards model and Fine Gray Regression model were used to investigate correlations between clinical and treatment factors with OS, LC and liver failure. All statistical analyses were performed using $\mathrm{R}$, version 3.6.1 (R Foundation), and a $\mathrm{P}<0.05$ was considered statistically significant.

\section{Results}

Forty-five patients underwent liver-direct RT for HCC at our institution, of whom 14 (31.1\%) received ablative RT. All patients had Karnofsky performance status (KPS) of $\geq 70$. The majority were males and had CP-A score. One patient had CP-C in the non-ablative RT group and had no other treatment option. The median tumor size in the ablative RT group was $3.8 \mathrm{~cm}(2.8-4.65 \mathrm{~cm})$ compared to a median of $8.1 \mathrm{~cm}(1.1-18.3 \mathrm{~cm})$ in the non-ablative group. Patients and tumor characteristics stratified by BED are summarized in Table 2.

More patients in the non-ablative RT group were pretreated with surgery or liver embolization prior to receiving $\mathrm{RT}$ than in the ablative $\mathrm{RT}$ group $(25.8 \%$ and $35.5 \%$ vs. $7.7 \%$ and $7.1 \%$, respectively). Use of systemic therapy prior to RT was similar in both groups $(12.9 \%$ and $14.3 \%$ ), with sorafenib as the drug of choice. Only one patient received doxorubicin and gemcitabine, in addition to sorafenib. Median nominal RT dose in the non-ablative RT group was $40 \mathrm{~Gy}$ in a median of 10 fractions with a median BED of $52.7 \mathrm{~Gy}$, compared to a median nominal dose of 60 Gy in a median of 12 fractions with a median BED of 96.9 Gy for the ablative RT group. Figure 1 illustrates isodose lines distribution of RT treatment for HCC showing an ablative RT course in (Figure 1A), 37.5 Gy/60 Gy in 15 fractions, and a non-ablative RT course in (Figure 1B), 30 Gy/40 Gy in 5 fractions. Treatment characteristics are summarized in Table 3.

When assessed at early follow-up (within 6 months of RT), patients treated with ablative RT had better radiographic responses compared to patients treated with BED $<80$ Gy, with $7 \%$ progressing versus $19.2 \%$, and $21.4 \%$ with complete response versus none $(\mathrm{P}=0.038)$ (Table 4).

The 1-year cumulative incidence of local tumor control for the whole cohort was $80.4 \%, 91.7 \%$ for ablative and $75.2 \%$ for BED <80 Gy. Hazard ratio (HR) for 1-year cumulative incidence of local tumor progression was 0.4 for the group treated with ablative $\mathrm{RT}(\mathrm{P}=0.22)$. Tumor size was not significantly associated with local progression (HR: $1.05, \mathrm{P}=0.38)$.

Liver failure possibly related to RT (within 3 months of RT and with no other identifiable cause) occurred in 4 patients, none in the ablative RT group (Table 5). Most other non-RT related liver failure cases were associated with local tumor progression on imaging ( $\mathrm{n}=7$ out of 12 cases). Out of 16 patients who developed liver failure, 15 patients had deterioration of their liver function with a change in CP score of two or more points (Table 6) and died within 3 months of liver failure.

On univariate analysis, CP score (HR: 3 for CP-B, 
Table 2 Patients and tumor characteristics stratified by BED

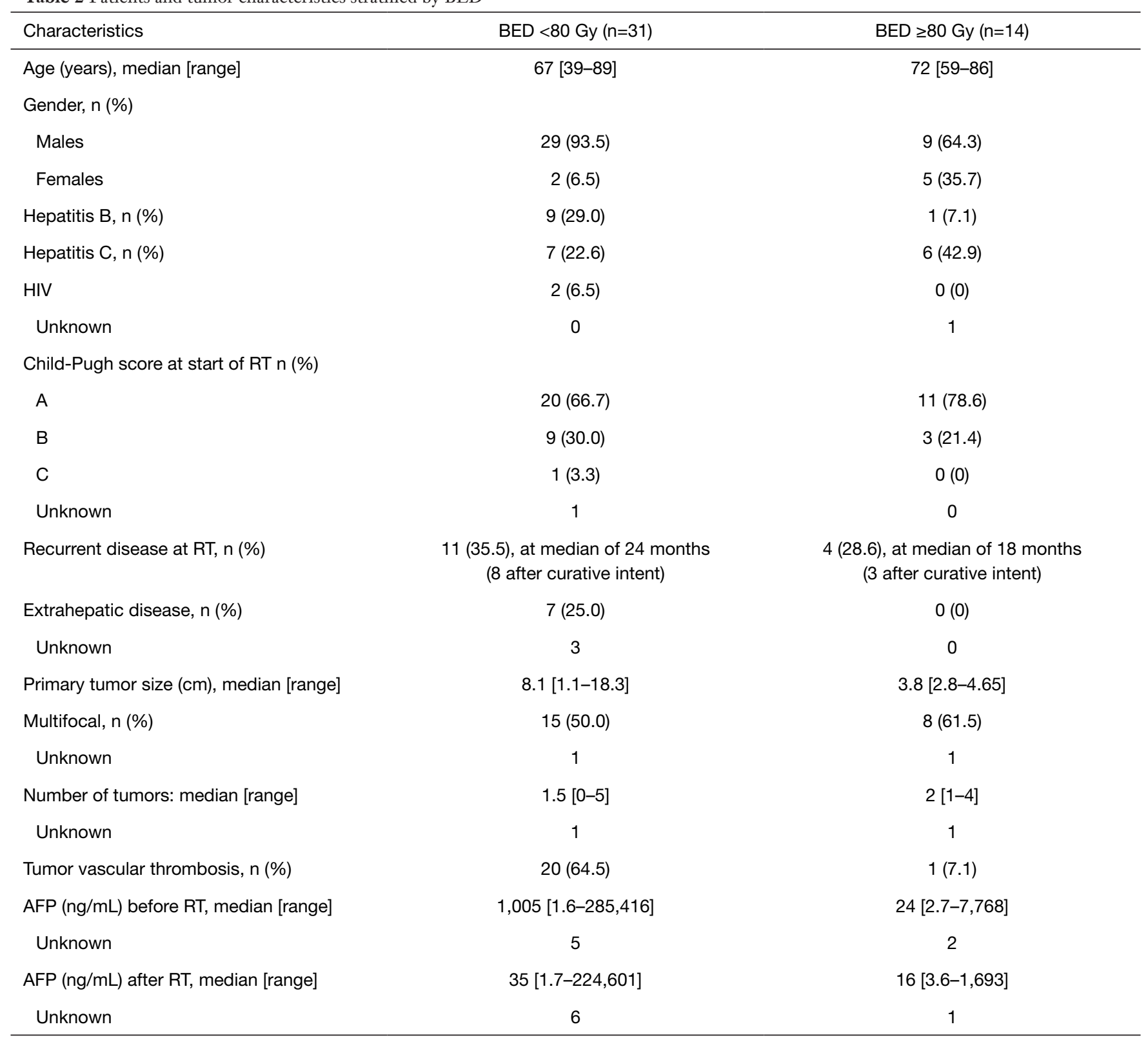

$\mathrm{BED}$, biologically effective dose; RT, radiation therapy; AFP, alpha fetoprotein.

HR: 16 for CP-C) and BED (HR: 7.69 for BED <80 Gy) correlated significantly with liver failure. There were too few events to run multivariate analysis of the above variables.

The 1-year cumulative incidence of distant progression was $54.1 \%$ (95\% CI: $37.0 \%, 68.5 \%$ ) with no difference by BED $(\mathrm{P}=0.76)$. Distant progression was distributed as follows: around $50 \%$ isolated in the liver, $15 \%$ involved the liver and other sites, and $35 \%$ extrahepatic.
With a median follow-up of 10.1 months, median overall survival (mOS) for the whole cohort was 9 months. On univariate analysis, only BED $\geq 80$ Gy was associated with better OS (HR: 0.4; $\mathrm{P}=0.044$ ). Tumor vascular thrombosis (HR: 1.85, $\mathrm{P}=0.09$ ), tumor size (HR: $1.05, \mathrm{P}=0.38$ ), multifocality (HR: 1.44, $\mathrm{P}=0.3$ ), number of liver tumors (HR: 1.12, $\mathrm{P}=0.3$ ), extrahepatic disease (HR: 1.1, $\mathrm{P}=0.8$ ), and $\mathrm{CP}$ score (HR: $1.91, \mathrm{P}=0.11$ ) were not significantly associated with OS. mOS and 1-year OS were 7 months and 

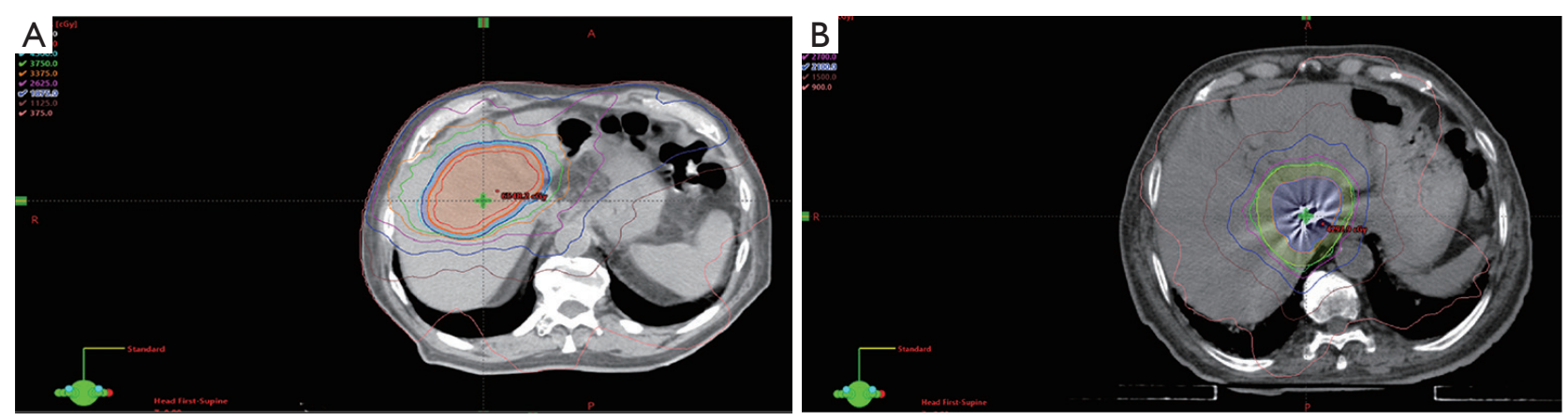

Figure 1 Isodose lines distribution of radiation therapy (RT) treatment for hepatocellular carcinoma (HCC) showing an ablative RT course in (A) 37.5 Gy/60 Gy in 15 fractions [planning target volume (PTV) 37.5 Gy in blue and PTV 60 Gy highlighted in orange] and a nonablative RT course in (B) 30 Gy/40 Gy in 5 fractions (PTV 40 Gy highlighted in blue and PTV 30 Gy in green).

Table 3 Treatment characteristics stratified by BED

\begin{tabular}{|c|c|c|}
\hline Treatments & BED $<80$ Gy $(n=31)$ & BED $\geq 80$ Gy $(n=14)$ \\
\hline Unknown & 0 & 1 \\
\hline Radiofrequency ablation before RT, n (\%) & 7 (22.5) [4 (12.9) to irradiated lesion] & 4 (28.6) [3 (21.4) to irradiated lesion] \\
\hline Embolization before RT, n (\%) & 11 (35.5) [5 (16.1) to irradiated lesion] & 1 (7.1) (0 to irradiated lesion) \\
\hline Prior $\mathrm{RT}^{\star}, \mathrm{n}(\%)$ & 0 & $1(7.1)^{\star}$ \\
\hline Unknown & 0 & 1 \\
\hline Systemic treatment before RT, n (\%) & $4(12.9)$ & $2(14.3)$ \\
\hline RT dose (Gy), median [range] & $40[20-62.5]$ & $60[50-75]$ \\
\hline
\end{tabular}

*, prior 55 Gy/25 fractions to primary liver HCC then developed regional (periportal) recurrence for which the patient received ablative RT. BED, biologically effective dose; RT, radiation therapy; HCC, hepatocellular carcinoma.

Table 4 RECIST response by BED, 3-6 months after end of RT $(\mathrm{P}=0.038)$

\begin{tabular}{lcc}
\hline RECIST response & $\begin{array}{c}\text { BED }<80 \text { Gy }(\mathrm{n}=31), \\
\mathrm{n}(\%)\end{array}$ & $\begin{array}{c}\mathrm{BED} \geq 80 \mathrm{~Gy}(\mathrm{n}=14), \\
\mathrm{n}(\%)\end{array}$ \\
\hline Progression & $5(19.2)$ & $1(7.1)$ \\
Stable & $9(34.6)$ & $7(50.0)$ \\
Partial response & $12(46.2)$ & $3(21.4)$ \\
Complete response & 0 & $3(21.4)$ \\
\hline
\end{tabular}

$\mathrm{BED}$, biologically effective dose; $\mathrm{RT}$, radiation therapy.
Table 5 The causes of liver failure/deterioration in liver function stratified by BED

\begin{tabular}{lcc}
\hline Liver failure & BED $<80$ Gy $(n=25)$ & BED $\geq 80$ Gy $(n=13)$ \\
\hline No failure & $10(40.0)$ & $12(92.3)$ \\
$\begin{array}{l}\text { Could be related to RT } \\
\text { (no other identifiable } \\
\text { causes) }\end{array}$ & $4(16.0)$ & $0(0)$ \\
Not related to RT $^{*}$ & $11(44.0)$ & $1(7.7)$
\end{tabular}

*, majority of the cases were due to disease progression in the liver. BED, biologically effective dose; RT, radiation therapy. 
Table 6 Change in CP score of patients who experienced liver failure within 3 months of death

\begin{tabular}{lc}
\hline Change in CP score & Count (\%) \\
\hline From CP-A to CP-B8 & $2(12.5)$ \\
From CP-A to CP-B9 & $3(18.8)$ \\
From CP-A to CP-C & $4(25.0)$ \\
From B7 to B9 & $1(6.3)$ \\
From CP-B7/8 to CP-C & $5(31.3)$ \\
From CP-C10 to CP-C11 & $1(6.3)$ \\
\hline
\end{tabular}

$\mathrm{CP}$, Child-Pugh.

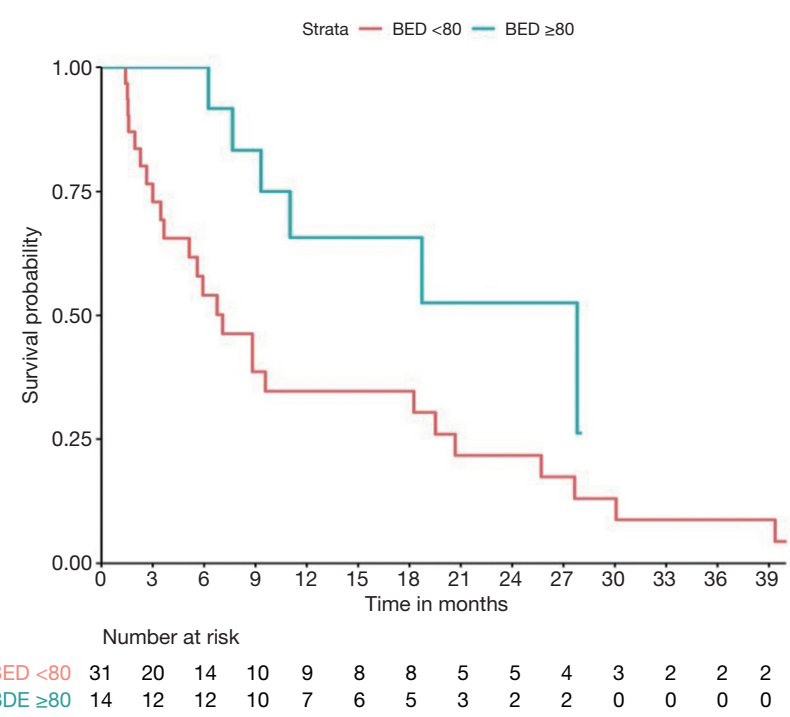

Figure 2 Overall survival (OS) from the time of radiation therapy (RT) stratified by biologically effective dose (BED) (<80 vs. $\geq 80$ Gy), $\mathrm{P}=0.044$.

$35 \%$ respectively for BED $<80$ Gy compared to 28 months and $66 \%$ for $\mathrm{BED} \geq 80$ Gy $(\mathrm{P}=0.044)$ (Figure 2). Median PFS was 3.4 months for BED $<80$ Gy vs. 7 months for BED $\geq 80$ Gy, but didn't reach statistical significance $(\mathrm{P}=0.06)$ (Figure 3).

Fatigue was the most common side effect of RT in both groups $(77.4 \%$ for BED $<80$ Gy and $78.6 \%$ for BED $\geq 80$ Gy) followed by CTCAE grade 2 or less ascites, thrombocytopenia and nausea $(50.0 \%$ vs. $28.6 \%, 41.9 \%$ vs. $57.1 \%$ and $22.6 \%$ vs. $14.3 \%$ for BED $<80$ Gy vs. BED $\geq 80$ Gy, respectively). As for grade 3+ toxicity that could be related to $\mathrm{RT}$, there were 3 patients who had ascites requiring drainage (by large volume paracentesis or

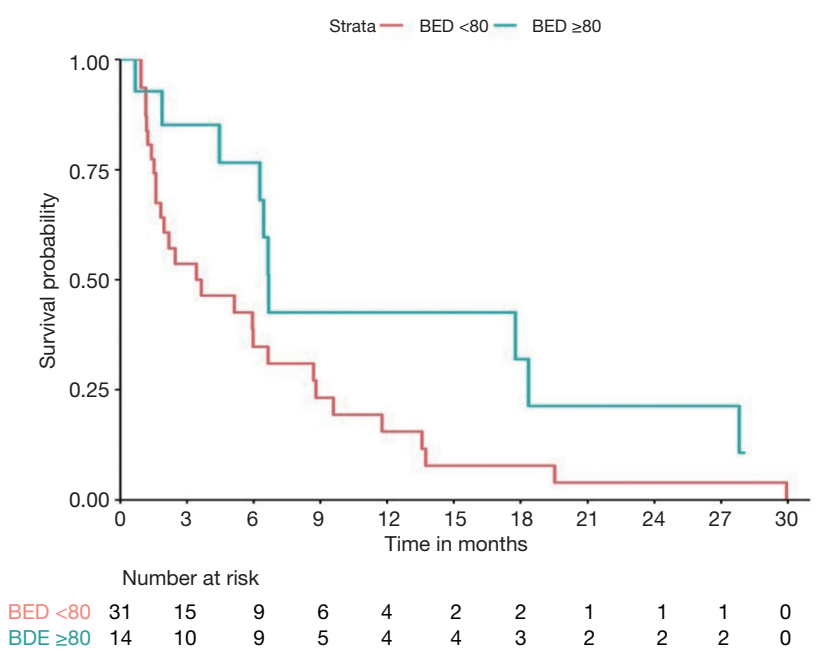

Figure 3 Progression-free survival (PFS) stratified by biologically effective dose (BED) (<80 vs. $\geq 80 \mathrm{~Gy}), \mathrm{P}=0.06$.

Tenckhoff catheter) only in the BED $<80$ Gy group. No grade $3+$ bowel toxicity was reported in either group (Table 7).

\section{Discussion}

The treatment of inoperable HCC with ablative RT, BED $\geq 80 \mathrm{~Gy}$, was associated with decreased early radiographic progression and less local tumor progression-related liver failure in our subgroup of patients.

RECIST response at early follow-up was clearly different among patients treated with ablative $v s$. non-ablative RT where more patients in the ablative group had a complete response and fewer patients had progression of disease compared to the non-ablative group. However, actuarial LC, although numerically better for ablative RT (HR: 0.4 for BED $\geq 80 \mathrm{~Gy}$ ), did not reach statistical significance. This could be due to the relatively small sample size with few events in the ablative RT group.

Our results suggest that the improvement in survival in the ablative RT subgroup compared to the non-ablative BED $<80$ Gy RT doses (mOS: 28 vs. 7 months, respectively) could be at least partly related to the lower observed frequency of tumor progression-related liver failure. Death in patients with unresectable HCC is often related to liver failure as a consequence of local tumor progression, mainly causing portal or biliary obstruction, or functional liver parenchymal loss (5). In an MDACC study on IHCC patients, death resulted from tumor-related liver failure in $89 \%$ of patients in whom the cause of death could be determined (7). 
Table 7 Most frequent toxicities of radiation therapy treatment stratified by BED

\begin{tabular}{lcc}
\hline Toxicities & BED <80 Gy $(\mathrm{n}=31)$ & BED $\geq 80$ Gy $(\mathrm{n}=14)$ \\
\hline Fatigue, $\mathrm{n}(\%)$ & $24(77.4)$ & $11(78.6)$ \\
Thrombocytopenia, $\mathrm{n}(\%)$ & $13(41.9)$ & $8(57.1)$ \\
Nausea, $\mathrm{n}(\%)$ & $7(22.6)$ & $2(14.3)$ \\
Ascites not requiring drainage, $\mathrm{n}(\%)$ & $15(50.0)$ & 0 \\
$\quad$ Unknown & 1 & $0(0)$ \\
Ascites requiring drainage, $\mathrm{n}(\%)$ & $3(9.7)$ & $0(0)$ \\
Grade 3+ bowel toxicity, $\mathrm{n}(\%)$ & $0(0)$ & $0(0)$ \\
Liver failure (could be related to RT), $\mathrm{n}(\%)$ & $4(16.0)$ & 0 \\
\hline
\end{tabular}

$\mathrm{BED}$, biologically effective dose; $\mathrm{RT}$, radiation therapy.

In sequential phase I and II trials by Bujold et al., SBRT with doses of 24 to $54 \mathrm{~Gy}$ in six fractions delivered to locally advanced HCC in patients with CP-A score resulted in LC of around $87 \%$ at 1 year. In their trial, SBRT dose was associated with LC and the reported median survival was around 17 months. However, they had around 30\% grade $\geq 3$ toxicity, including seven patients whose death was possibly related to treatment (13). Another study on HCC patients with portal vein thrombosis, a retrospective analysis of SBRT versus conventionally fractionated RT (CVRT), showed that the use of SBRT and BED $\geq 65$ Gy were favorable predictors of survival. The 1-year OS was $34.9 \%$ for SBRT vs. $15.3 \%$ for CVRT. Their reported survival rate is lower than the 1-year OS of around $66 \%$ for our subgroup of patients treated with BED $\geq 80$ Gy. However, of note is that they used a lower BED and all of their patients had tumor vascular thrombosis, which may have contributed to inferior outcomes (17).

Compared to conventional RT, higher doses of ablative RT improve outcomes for inoperable HCC patients. Our most commonly used ablative doses were $50 \mathrm{~Gy}$ in 5 fractions or $60 \mathrm{~Gy}$ in 15 fractions. Ablative RT doses can be achieved with 3-6 fractions when doses approach around $54 \mathrm{~Gy}(5)$. In a review by Gerum et al. that included several retrospective and prospective phase I/II trials, SBRT (3-6 fractions) achieved good LC, $65-100 \%$ at 1 year (6). However, doses have to be reduced whenever safety is a concern mainly in tumors close to GI structures or the biliary tree which leads to decreased efficacy. The need for limiting toxicity and improving the therapeutic ratio remains essential for safe dose escalation. A more protracted hypofractionated ablative RT offers the advantage of increasing BED to the tumor while limiting toxicity to adjacent OAR.

For HCC, several hypofractionated RT trials have been published with ablative doses achieving high rates of LC and survival, but mainly using protons RT $(8-11,18)$. Hong et al. used hypofractionated RT for liver tumors, using protons, in a multi-institutional phase II trial that included 44 patients with localized inoperable HCC (8). They showed very good survival outcomes with median OS of 49.9 months (95\% CI lower bound, 17.8 months; upper bound not reached) and 1-year OS of 76.5\%.

Our study showed good local tumor control and a high rate of survival in patients treated with BED $\geq 80$ Gy using stereotactic hypofractionated photon RT which is more readily available than protons in most centers. IMRT was used for sparing of adjacent GI organs, with no grade 3+ bowel toxicity and no liver failure related to RT in the ablative subgroup.

It is important to note that OS in our subgroup of ablative RT is possibly confounded by other factors including patient selection, liver disease, distant metastasis, and a different era of treatment. The main limitation is that the two groups treated with ablative $v s$. non-ablative RT were different in terms of more extrahepatic disease, more advanced liver disease, more tumor vascular thrombosis, and larger median tumor sizes in the non-ablative RT compared to the ablative RT group. Another limitation is our inability to further subdivide patients with CP-B score due to their low number. Although those factors were not significantly associated with survival on univariate analysis, the retrospective design of our study with its inherent limitations does not allow for the same elimination of selection bias as a randomized controlled trial 
(RCT) would.

However, this sheds light on the importance of patient selection for ablative RT whereby this subgroup has been carefully selected to have no extrahepatic disease and good liver functional reserve, with no patients with $\mathrm{CP}$ score of B8 or higher.

There is an ongoing clinical trial randomizing patients with unresectable or locally recurrent HCC for photon versus proton RT in 5 or 15 fractions (ClinicalTrials.gov Identifier: NCT03186898). Our institution has partnered with a proton center and there's a plan to open the mentioned ongoing randomized trial for the treatment for HCC at our institution. The patients selected for ablative RT at the multidisciplinary tumor board should have unresectable or locally recurrent HCC, no extrahepatic disease (defined as extrahepatic metastases or malignant nodes $>3.0 \mathrm{~cm}$, in sum of maximal diameters), CP score of $\mathrm{A}$ or $\mathrm{B} 7$, and 3 or fewer single or multinodular tumors. This will provide more definitive results on the use of ablative photons or protons RT for HCC.

\section{Conclusions}

In conclusion, stereotactic ablative RT led to minimal toxicity, achieved $>90 \%$ LC, minimized tumor- and treatment-related liver failure, and was associated with improved survival (28 vs. 7 months, $\mathrm{P}=0.044$ ) compared to tumors treated with lower BED for highly selected inoperable HCC. It should be considered in well-selected patients with localized unresectable or locally recurrent HCC, in centers where it could be safely delivered.

\section{Acknowledgments}

This study was presented as a poster presentation at the American Society for Radiation Oncology (ASTRO) 2020 meeting.

Funding: None.

\section{Footnote}

Reporting Checklist: The authors have completed the STROBE reporting checklist. Available at https://dx.doi. org/10.21037/jgo-21-116

Data Sharing Statement: Available at https://dx.doi. org/10.21037/jgo-21-116
Peer Review File: Available at https://dx.doi.org/10.21037/ jgo-21-116

Conflicts of Interest: All authors have completed the ICMJE uniform disclosure form (available at https://dx.doi. org/10.21037/jgo-21-116). The authors have no conflicts of interest to declare

Ethical Statement: The authors are accountable for all aspects of the work in ensuring that questions related to the accuracy or integrity of any part of the work are appropriately investigated and resolved. The study was conducted in accordance with the Declaration of Helsinki (as revised in 2013). The study was approved by institutional board of Memorial Sloan Kettering Cancer Center (MSKCC), New York (IRB 16-370) and individual consent for this retrospective analysis was waived.

Open Access Statement: This is an Open Access article distributed in accordance with the Creative Commons Attribution-NonCommercial-NoDerivs 4.0 International License (CC BY-NC-ND 4.0), which permits the noncommercial replication and distribution of the article with the strict proviso that no changes or edits are made and the original work is properly cited (including links to both the formal publication through the relevant DOI and the license). See: https://creativecommons.org/licenses/by-nc-nd/4.0/.

\section{References}

1. Tapper EB, Parikh ND. Mortality due to cirrhosis and liver cancer in the United States, 1999-2016: observational study. BMJ 2018;362:k2817.

2. Gholam PM, Iyer R, Johnson MS. Multidisciplinary Management of Patients with Unresectable Hepatocellular Carcinoma: A Critical Appraisal of Current Evidence. Cancers (Basel) 2019;11:873.

3. Tabrizian P, Jibara G, Shrager B, et al. Recurrence of hepatocellular cancer after resection: patterns, treatments, and prognosis. Ann Surg 2015;261:947-55.

4. Yau T, Park JW, Finn RS, et al. CheckMate 459: A randomized, multi-center phase III study of nivolumab (NIVO) vs sorafenib (SOR) as first-line (1L) treatment in patients (pts) with advanced hepatocellular carcinoma (aHCC). Ann Oncol 2019;30:v874-5.

5. Reyngold M, Koay EJ, Crane CH. Hypofractionated ablative radiation therapy for hepatocellular carcinoma: 
practical considerations and review of the literature.

Hepatoma Res 2018;4:49.

6. Gerum S, Jensen AD, Roeder F. Stereotactic body radiation therapy in patients with hepatocellular carcinoma: A mini-review. World J Gastrointest Oncol 2019;11:367-76.

7. Tao R, Krishnan S, Bhosale PR, et al. Ablative Radiotherapy Doses Lead to a Substantial Prolongation of Survival in Patients With Inoperable Intrahepatic Cholangiocarcinoma: A Retrospective Dose Response Analysis. J Clin Oncol 2016;34:219-26.

8. Hong TS, Wo JY, Yeap BY, et al. Multi-Institutional Phase II Study of High-Dose Hypofractionated Proton Beam Therapy in Patients with Localized, Unresectable Hepatocellular Carcinoma and Intrahepatic Cholangiocarcinoma. J Clin Oncol 2016;34:460-8.

9. Kawashima M, Furuse J, Nishio T, et al. Phase II study of radiotherapy employing proton beam for hepatocellular carcinoma. J Clin Oncol 2005;23:1839-46.

10. Bush DA, Kayali Z, Grove R, et al. The safety and efficacy of high-dose proton beam radiotherapy for hepatocellular carcinoma: a phase 2 prospective trial. Cancer 2011;117:3053-9.

11. Mizumoto M, Okumura T, Hashimoto T, et al. Proton beam therapy for hepatocellular carcinoma: a comparison of three treatment protocols. Int J Radiat Oncol Biol Phys 2011;81:1039-45.

12. Scorsetti M, Comito T, Clerici E, et al. Phase II trial

Cite this article as: Hilal L, Reyngold $\mathrm{M}, \mathrm{Wu}$ AJ, Araji A, Abou-Alfa GK, Jarnagin W, Harding JJ, Gambarin M, El Dika I, Brady P, Navilio J, Berry SL, Flynn J, Zhang Z, Tuli R, Zinovoy M, Romesser PB, Cuaron JJ, Crane CH, Hajj C. Ablative radiation therapy for hepatocellular carcinoma is associated with reduced treatment- and tumor-related liver failure and improved survival. J Gastrointest Oncol 2021;12(4):1743-1752. doi: 10.21037/jgo-21-116 on SBRT for unresectable liver metastases: long-term outcome and prognostic factors of survival after 5 years of follow-up. Radiat Oncol 2018;13:234.

13. Bujold A, Massey CA, Kim JJ, et al. Sequential phase I and II trials of stereotactic body radiotherapy for locally advanced hepatocellular carcinoma. J Clin Oncol 2013;31:1631-9.

14. Pugh RN, Murray-Lyon IM, Dawson JL, et al. Transection of the oesophagus for bleeding oesophageal varices. $\mathrm{Br} \mathrm{J}$ Surg 1973;60:646-9.

15. Eisenhauer EA, Therasse P, Bogaerts J, et al. New response evaluation criteria in solid tumours: revised RECIST guideline (version 1.1). Eur J Cancer 2009;45:228-47.

16. Cancer Therapy Evaluation Program (CTEP). Common Terminology Criteria for Adverse Events (CTCAE). Version 5.0 [5x7]. U.S. Department of Health And Human Services, 2017.

17. Yang JF, Lo CH, Lee MS, et al. Stereotactic ablative radiotherapy versus conventionally fractionated radiotherapy in the treatment of hepatocellular carcinoma with portal vein invasion: a retrospective analysis. Radiat Oncol 2019;14:180.

18. Chadha AS, Gunther JR, Minsky BD, et al. Proton Therapy Outcomes for Localized, Unresectable Hepatocellular Carcinoma. Int J Radiat Oncol Biol Phys 2016;96:e181-2. 